\title{
Probability Densities for Fluorescent Photons Emitted by a Two-State Atom Driven by a Laser
}

\author{
Robertsen A. Riehle and Henk F. Arnoldus \\ Department of Physics and Astronomy, Mississippi State University, P.O. Drawer 5167, Mississippi State, MS 39762-5167, USA \\ Correspondence should be addressed to Henk F. Arnoldus, hfa1@msstate.edu
}

Received 20 September 2012; Accepted 14 November 2012

Academic Editors: J. M. Girkin, D. Poitras, and M. Potasek

Copyright ( $) 2012$ R. A. Riehle and H. F. Arnoldus. This is an open access article distributed under the Creative Commons Attribution License, which permits unrestricted use, distribution, and reproduction in any medium, provided the original work is properly cited.

Fluorescent photons emitted by a two-state atom in a laser beam are correlated. We have obtained the probability density for the emission of the $n$th photon after a random initial time $t=0$. It is shown that the correlations between the photons lead to a deviation from the poissonian value for this function (the probability density for independent events), although the deviation is not as significant as one may expect.

\section{Introduction}

When a two-state atom, with energy level separation $\hbar \omega_{0}$, is immersed in a laser beam with an angular frequency $\omega_{L}$, then photons are exchanged between the atom and the field in stimulated absorption and emission, provided that $\omega_{L}$ is near $\omega_{o}$. We shall consider the case of resonance, so $\omega_{o}=\omega_{L}$. In addition, photons are emitted in spontaneous transitions from the excited state to the ground state, and these photons are emitted in all direction as electric dipole radiation. The emission of these fluorescent photons can be considered as a random event process on the time axis, and we assume that the duration of each event is negligible (dots on the time axis). This interpretation can be justified with the theory of photon detection from an electromagnetic field $[1,2]$. Let $A$ be the Einstein coefficient for spontaneous transitions from the excited state to the ground state [3] and let $n_{e}$ be the population of the excited state. Then, the number of emitted photons per second is equal to

$$
I=A n_{e}
$$

and this is the intensity of the random process. We shall assume that the atom is in the steady state, so that $I$ is independent of time. The temporal statistics of photon emissions can be represented by the probability densities $w_{n}(t)$, with $n=1,2, \ldots$. Let $\tau_{n}$ be the time at which the $n$th photon is emitted, after an initial time $t=0$. Since photons are emitted randomly, $\tau_{n}$ is a random variable, and its probability density is $w_{n}(t)$ :

$$
w_{n}(t) \mathrm{d} t=\text { probability that } \tau_{n} \text { lies in }[t, t+\mathrm{d} t] .
$$

We shall evaluate $w_{n}(t)$ for the emission of fluorescent photons.

If photons were emitted independently of each other, then the emission process would be a Poisson process, and the probability for the emission of $n$ photons in $[0, t]$ would be

$$
P_{n}(t)=\frac{(I t)^{n}}{n !} e^{-I t} .
$$

The probability for the emission of the $n$th photon in $[t, t+$ $\mathrm{d} t]$ is $I \mathrm{~d} t$. If this emission would be independent of previous emissions, then

$$
w_{n}(t) \mathrm{d} t=P_{n-1}(t) I \mathrm{~d} t
$$

so that

$$
w_{n}(t)=I \frac{(I t)^{n-1}}{(n-1) !} e^{-I t}
$$




\section{Photon Correlations in Resonance Fluorescence}

Correlations between random events are expressed through the intensity correlation functions $[4,5]$ :

$$
\begin{aligned}
I_{k}\left(t_{1}, t_{2}, \ldots, t_{k}\right) \mathrm{d} t_{1} \mathrm{~d} t_{2} \cdots \mathrm{d} t_{k} \\
=\text { probability for a photon emission in }\left[t_{1}, t_{1}+\mathrm{d} t_{1}\right], \\
\quad \text { and... and a photon emission in }\left[t_{k}, t_{k}+\mathrm{d} t_{k}\right], \\
\quad \text { irrespective of emissions at other times, and with } \\
\\
t_{1}<t_{2}<\cdots<t_{k} .
\end{aligned}
$$

For resonance fluorescence, these correlation functions take the form $[6,7]$

$$
\begin{array}{r}
I_{k}\left(t_{1}, \ldots, t_{k}\right)=A^{k} f\left(t_{k}-t_{k-1}\right) \cdots \\
\quad f\left(t_{2}-t_{1}\right) n_{e}, \\
k=2,3, \ldots,
\end{array}
$$

involving the function $f(t)$. This function equals the population of the excited state at time $t$, under the condition that the atom is in the ground state at $t=0$. Therefore,

$$
f(0)=0 .
$$

From (7), we then see that an intensity correlation function vanishes when two consecutive time arguments are the same. Consequently, immediately after the emission of a photon, there can be no emission of the next photon. This phenomenon is called antibunching and has been observed experimentally [8-10]. Physically, this can be understood from the fact that after the emission of a photon the atom is in the ground state, whereas a photon can only be emitted when the atom is in the excited state. It takes a finite time for the atom to make the transition from the ground state to the excited state, and, therefore, the probability for an emission immediately after a previous one is zero. We also have

$$
f(\infty)=n_{e},
$$

since $n_{e}$ is the population of the excited state in the steady state.

The function $f(t)$ can be evaluated by solving the equation of motion for the system. The free parameter is the the Rabi frequency $\Omega$, which is assumed to be positive. This parameter depends on the atomic transition dipole moment, and $\Omega^{2}$ is proportional to the power of the laser. We find that [11]

$$
f(t)=n_{e}\left\{1-e^{-(3 / 4) \hat{t}}\left[\frac{3}{4 \rho} \sinh (\rho \hat{t})+\cosh (\rho \hat{t})\right]\right\},
$$

where we have set $\hat{t}=A t$ for the time in units of the life time $1 / A$ of the excited state. The parameter $\rho$ is defined as

$$
\rho=\sqrt{\frac{1}{16}-\hat{\Omega}^{2}}
$$

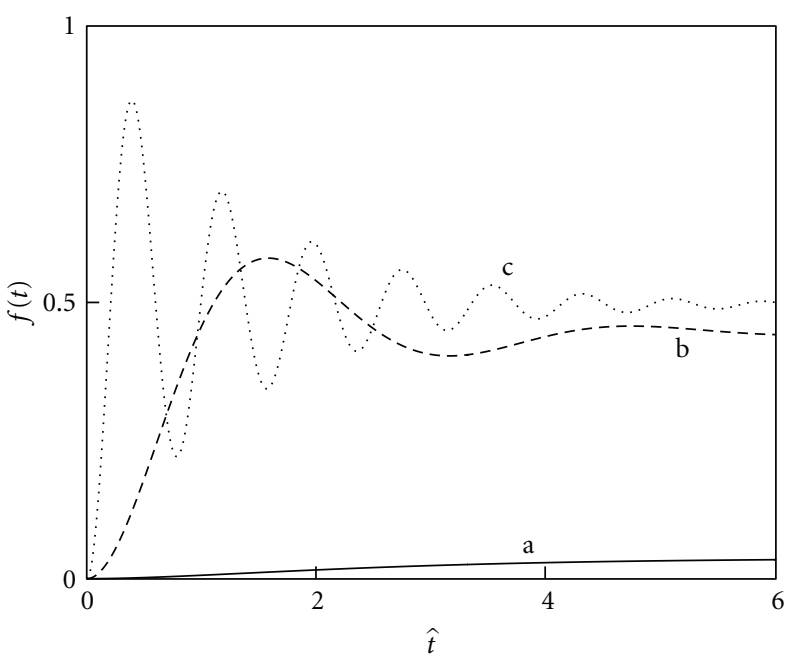

FIGURE 1: The graph shows the function $f(t)$ for $\widehat{\Omega}=0.2$ (curve a), $\widehat{\Omega}=2$ (curve $\mathrm{b}$ ), and $\hat{\Omega}=8$ (curve c). For small $\hat{\Omega}$, the function is exponential, and for large $\widehat{\Omega}$ the function is oscillatory.

with $\hat{\Omega}=\Omega / A$ being the Rabi frequency in units of $A$. For $\hat{\Omega}<1 / 4$, the function $f(t)$ reaches its steady state $n_{e}$ exponentially, but for $\hat{\Omega}>1 / 4$, the hyperbolic functions become trigonometric functions, and oscillations appear. Figure 1 illustrates the behavior of $f(t)$. The steady state is found to be

$$
n_{e}=\frac{\widehat{\Omega}^{2}}{1+2 \widehat{\Omega}^{2}} .
$$

\section{Laplace Transform of the Probability Densities}

The intensity correlation functions $I_{k}\left(t_{1}, \ldots, t_{k}\right)$ contain all information about the random process, and the probability densities can be found from these functions [12]. It appears convenient to adopt a Laplace transform of $w_{n}(t)$ :

$$
\widetilde{w}_{n}(s)=\int_{0}^{\infty} e^{-s t} w_{n}(t) \mathrm{d} t .
$$

We then obtain that [13]

$$
\widetilde{w}_{n}(s \mid 0)=\frac{I}{s[1+A \tilde{f}(s)]}\left(\frac{A \tilde{f}(s)}{1+A \tilde{f}(s)}\right)^{n-1},
$$

in terms of the Laplace transform $\tilde{f}(s)$ of $f(t)$.

From (10), we find that

$$
\tilde{f}(s)=\frac{\Omega^{2}}{2 s} \frac{1}{(A+s)((1 / 2) A+s)+\Omega^{2}},
$$


and this yields

$$
\begin{aligned}
\tilde{w}_{n}(s \mid 0)= & I\left((1 / 2) A \Omega^{2}\right)^{n-1} \\
& \times \frac{(s+A)(s+(1 / 2) A)+\Omega^{2}}{\left\{(s+(1 / 2) A)\left[(s+(1 / 2) A)^{2}-\gamma^{2} A^{2}\right]\right\}^{n}},
\end{aligned}
$$

where we have set

$$
\gamma=\sqrt{\frac{1}{4}-\hat{\Omega}^{2}} .
$$

\section{Probability Densities}

In order to evaluate the Laplace inverse of (16), we first shift the parameter $s$ with the attenuation theorem:

$$
w_{n}(t)=I\left(\frac{1}{2} A \Omega^{2}\right)^{n-1} e^{-(1 / 2) A t} \mathcal{L}^{-1}\left\{\frac{s(s+(1 / 2) A)+\Omega^{2}}{s^{n}\left[s^{2}-(A \gamma)^{2}\right]^{n}}\right\} .
$$

The inverse on the right-hand side can be computed with the Bromwich integral [14]. We find that

$$
\begin{aligned}
w_{n}(t)= & I\left(\frac{1}{8} \hat{\Omega}^{2}\right)^{n-1} \frac{1}{\gamma^{3 n-1}} e^{-(1 / 2) \hat{t}} \\
\times & \sum_{k=0}^{n-1} \sum_{r=0}^{n-1-k} \frac{(\gamma \hat{t})^{n-1-k-r}}{(n-1-k-r) !} \\
\times & \left\{\frac{1}{2}\left[(-1)^{k} v_{r}(\gamma) e^{\gamma \hat{t}}+v_{r}(-\gamma) e^{-\gamma \hat{t}}\right]\right. \\
& \left.\times A_{n-1, k}-v_{r}(0) B_{n-1, k}\right\} .
\end{aligned}
$$

The functions $v_{r}(x)$ are defined as

$$
\begin{gathered}
v_{0}(x)=x\left(x+\frac{1}{2}\right)+\widehat{\Omega}^{2}, \\
v_{1}(x)=\gamma\left(2 x+\frac{1}{2}\right), \\
v_{2}(x)=\gamma^{2}, \\
v_{r}(x)=0, \quad r>2,
\end{gathered}
$$

and the coefficients $A_{n, k}$ and $B_{n, k}$ are

$$
\begin{gathered}
A_{n, k}=\sum_{m=0}^{k}\left(\begin{array}{c}
n+m \\
n
\end{array}\right)\left(\begin{array}{c}
n+k-m \\
n
\end{array}\right) 2^{n-m}, \\
B_{n, k}=2^{2 n} \sum_{m=0}^{k}\left(\begin{array}{c}
n+m \\
n
\end{array}\right)\left(\begin{array}{c}
n+k-m \\
n
\end{array}\right)(-1)^{n-m},
\end{gathered}
$$

for $n=0,1, \ldots$, and $k=0,1, \ldots$ A variety of properties of these coefficients have been derived elsewhere [15]. For small $n$ and $k$, these coefficients can be computed from (21), and
TABLe 1: Table of $A_{n, k}$.

\begin{tabular}{ccccc}
\hline$n$ & & \multicolumn{3}{c}{$k$} \\
& 0 & 1 & 2 & 3 \\
\hline 0 & 1 & $3 / 2$ & $7 / 4$ & $15 / 8$ \\
1 & 2 & 6 & $23 / 2$ & 18 \\
2 & 4 & 18 & 48 & 99 \\
3 & 8 & 48 & 164 & 420 \\
\hline
\end{tabular}

TABle 2: Table of $B_{n, k}$.

\begin{tabular}{ccccc}
\hline$n$ & & & $k$ & \\
& 0 & 1 & 2 & 3 \\
\hline 0 & 1 & 0 & 1 & 0 \\
1 & -4 & 0 & -8 & 0 \\
2 & 16 & 0 & 48 & 0 \\
3 & -64 & 0 & -256 & 0 \\
\hline
\end{tabular}

the results are tabulated in Tables 1 and 2 . For $t=0$, only the term with $r=n-1-k$ survives, and with various sumrules for the coefficients $A_{n, k}$ and $B_{n, k}$, we derive the initial values

$$
w_{n}(0)=I \delta_{n, 1} .
$$

On general grounds, this relation holds for any probability density of a random event process.

\section{Deviation from Poisson Statistics}

The expression on the right-hand side of (19) for $w_{n}(t)$ is the main result of this paper. From this rather formidable formula, it is not readily clear what the significance of this result is. In order to discover the main features of the probability densities, we work out the cases for $n=1$ and $n=2$. We find that

$$
\begin{gathered}
w_{1}(t)=I \frac{1}{\gamma^{2}} e^{-(1 / 2) \hat{t}}\left[-\hat{\Omega}^{2}+\frac{1}{2} \gamma \sinh (\gamma \hat{t})+\frac{1}{4} \cosh (\gamma \hat{t})\right], \\
w_{2}(t)=I \frac{\widehat{\Omega}^{2}}{8 \gamma^{4}} e^{-(1 / 2) \hat{t}}\left[2+4 \hat{\Omega}^{2} \hat{t}+\left(4 \gamma-\frac{3}{2 \gamma}+\gamma \hat{t}\right) \sinh (\gamma \hat{t})\right. \\
\left.+\left(\frac{1}{2} \hat{t}-2\right) \cosh (\gamma \hat{t})\right] .
\end{gathered}
$$

Photons are emitted randomly, but the photons are correlated, as can be seen from Figure 1. For uncorrelated photons, the function $f(t)$ would be a constant: $f(t)=f(\infty)=$ $n_{e}$, and $w_{n}(t)$ would be the Poisson distribution given by (5) (with $I=A n_{e}$ ). The solid curves in Figures 2, 3, and 4 show $w_{1}(t)$ for $\hat{\Omega}=0.2,2$, and 8 , respectively, and the dashed curves are $w_{1}(t)$ for a Poisson distribution with the same intensity. These parameters are the same as for the various functions $f(t)$ in Figure 1. We see from Figure 2 that for small laser power the probability density for the emitted photons is almost indistinguishable from the Poisson distribution. For larger $\widehat{\Omega}$, oscillations set in, and $w_{1}(t)$ deviates more 


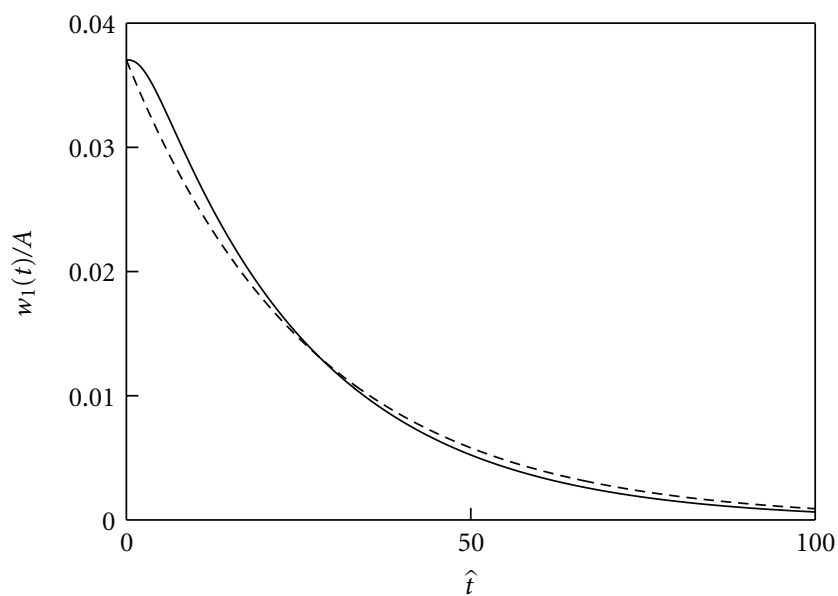

Figure 2: The graph shows $w_{1}(t) / A$ for $\widehat{\Omega}=0.2$.

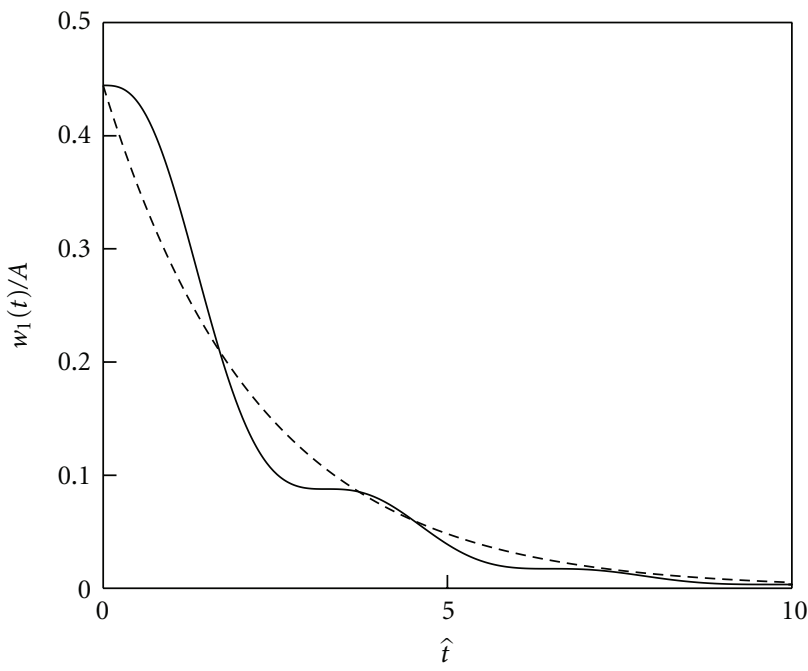

FIgUre 3: The graph shows $w_{1}(t) / A$ for $\widehat{\Omega}=2$.

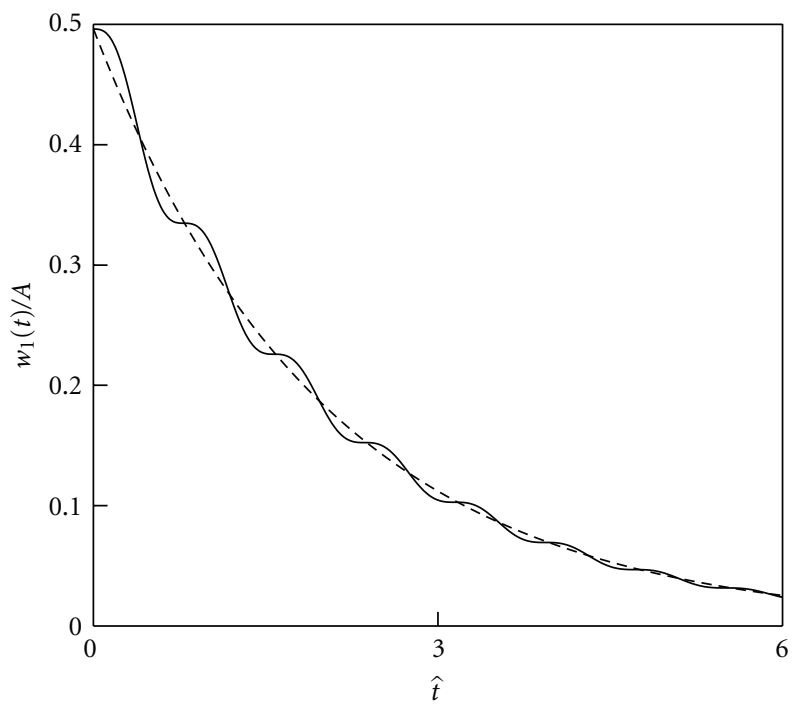

Figure 4: The graph shows $w_{1}(t) / A$ for $\widehat{\Omega}=8$. 


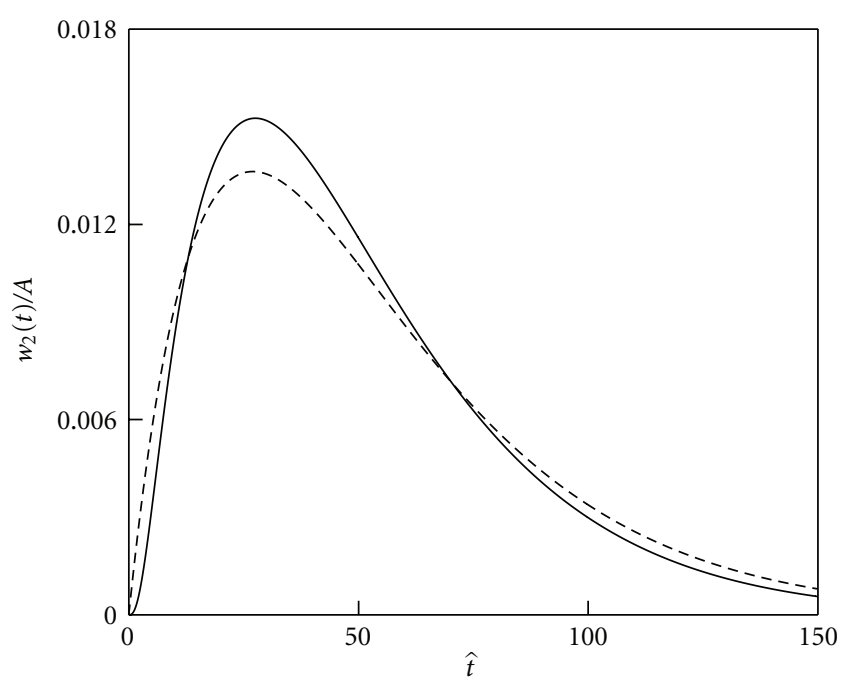

FIgURE 5: The graph shows $w_{2}(t) / A$ for $\widehat{\Omega}=0.2$.

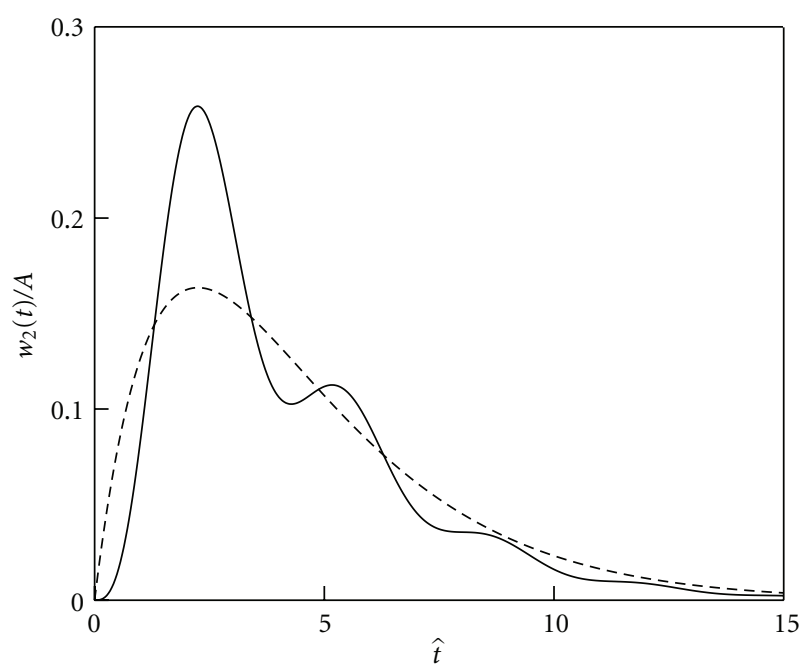

FIGURE 6: The graph shows $w_{2}(t) / A$ for $\widehat{\Omega}=2$.

noticeably from the Poisson curve, as illustrated in Figure 3. For even larger $\hat{\Omega}$, as in Figure 4, the oscillations get faster, but the amplitude of the oscillations diminishes, and the probability density is again nearly poissonian. The probability density in Figure 4 is determined by curve $c$ for $f(t)$ in Figure 1, and this function deviates very strongly from its Poisson value $f(\infty)$. We notice that the strong oscillations in $f(t)$ result only in small ripples on the function $w_{1}(t)$ in Figure 4, which appears quite remarkable. Figures 5-7 show $w_{2}(t)$ for the same values of $\widehat{\Omega}$ as in Figures 2-4. It appears that $w_{2}(t)$ deviates more from Poisson statistics, in particular for moderate laser power, as in Figure 6.

\section{Conclusions}

A two-state atom in a laser beam emits fluorescent photons. We have obtained the probability density $w_{n}(t)$ for the emission of the $n$th photon after a random initial time $t=0$. It is assumed that the laser frequency is on resonance with

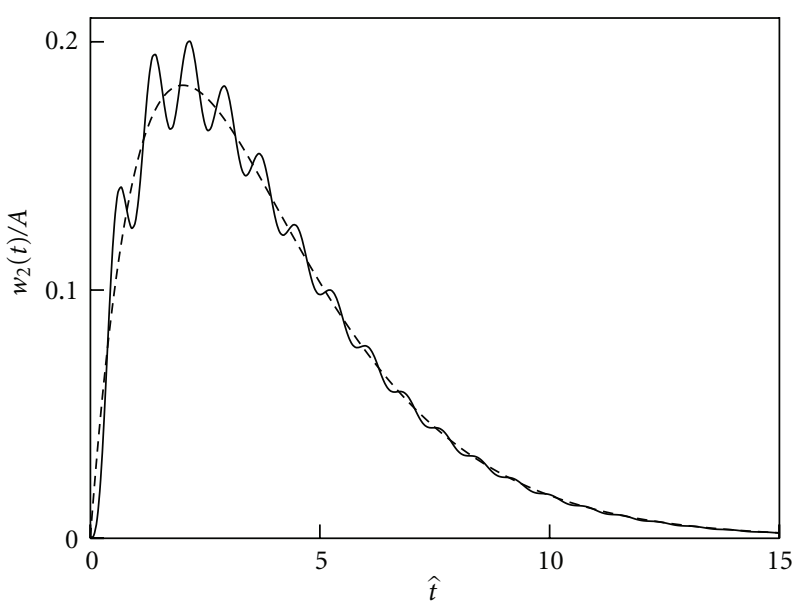

FIgURE 7: The graph shows $w_{2}(t) / A$ for $\widehat{\Omega}=8$.

the atomic transition. The result is given by (19), and it only has the dimensionless Rabi frequency $\widehat{\Omega}$ as free parameter. Of particular interest is the deviation from the probability density for independent events (Poisson process), since this reflects the correlations between emitted photons. The results are illustrated in Figures 2-7, and we observe that the functions $w_{n}(t)$ only deviate moderately from the corresponding functions for independent photon emissions.

\section{References}

[1] R. J. Glauber, "Optical coherence and photon statistics," in Quantum Optics and Electronics, C. DeWitt, A. Blandin, and C. Cohen-Tannoudji, Eds., pp. 65-185, Gordon and Breach, New York, NY, USA, 1965.

[2] P. L. Kelley and W. H. Kleiner, "Theory of electromagnetic field measurement and photoelectron counting," Physical Review, vol. 136, no. 2A, pp. A316-A334, 1964.

[3] R. Loudon, The Quantum Theory of Light, Clarendon Press, Oxford, UK, 2nd edition, 1983.

[4] R. L. Stratonovich, Topics in the Theory of Random Noise, vol. 1, chapter 6, Gordon and Breach, New York, NY, USA, 1963.

[5] N. G. van Kampen, Stochastic Processes in Physics and Chemistry, chapter 2, Elsevier, Amsterdam, The Netherlands, 3rd edition, 2007.

[6] G. S. Agarwal, "Time factorization of the higher-order intensity correlation functions in the theory of resonance fluorescence," Physical Review A, vol. 15, no. 2, pp. 814-816, 1977.

[7] D. Lenstra, "Photon-number statistics in resonance fluorescence," Physical Review A, vol. 26, no. 6, pp. 3369-3377, 1982.

[8] H. J. Kimble, M. Dagenais, and L. Mandel, "Photon antibunching in resonance fluorescence," Physical Review Letters, vol. 39, no. 11, pp. 691-695, 1977.

[9] M. Dagenais and L. Mandel, "Investigation of two-time correlations in photon emissions from a single atom," Physical Review A, vol. 18, no. 5, pp. 2217-2228, 1978.

[10] F. Diedrich and H. Walther, "Nonclassical radiation of a single stored ion," Physical Review Letters, vol. 58, no. 3, pp. 203-206, 1987. 
[11] D. F. Walls and G. J. Milburn, Quantum Optics, Springer, Berlin, Germany, 1994, p. 221.

[12] H. F. Arnoldus and R. A. Riehle, "Waiting times, probabilities and the Q factor of fluorescent photons," Journal of Modern Optics, vol. 59, no. 11, pp. 1002-1015, 2012.

[13] H. F. Arnoldus and G. Nienhuis, "Photon statistics of fluorescence radiation," Optica Acta, vol. 33, no. 6, pp. 691-702, 1986.

[14] G. B. Arfken and H. J. Weber, Mathematical Methods for Physicists, Academic Press, San Diego, Calif., USA, 4th edition, 1995.

[15] H. F. Arnoldus and R. A. Riehle, "Conditional probability densities for photon emission in resonance fluorescence," Physics Letters A, vol. 376, pp. 2584-2587, 2012. 

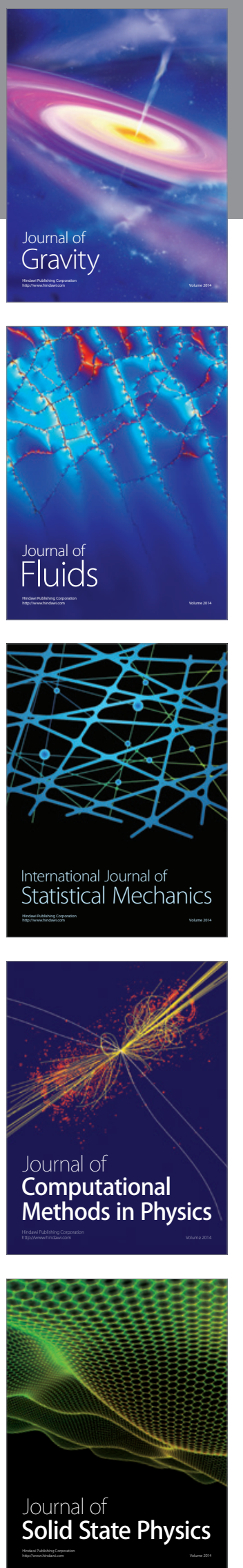
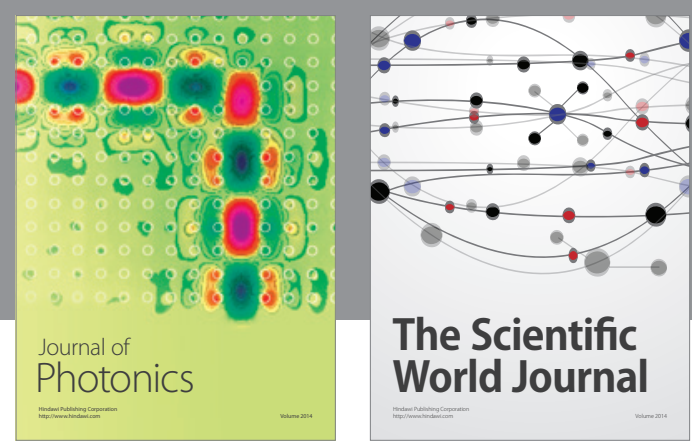

The Scientific World Journal

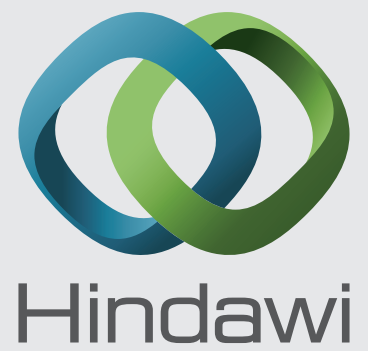

Submit your manuscripts at http://www.hindawi.com
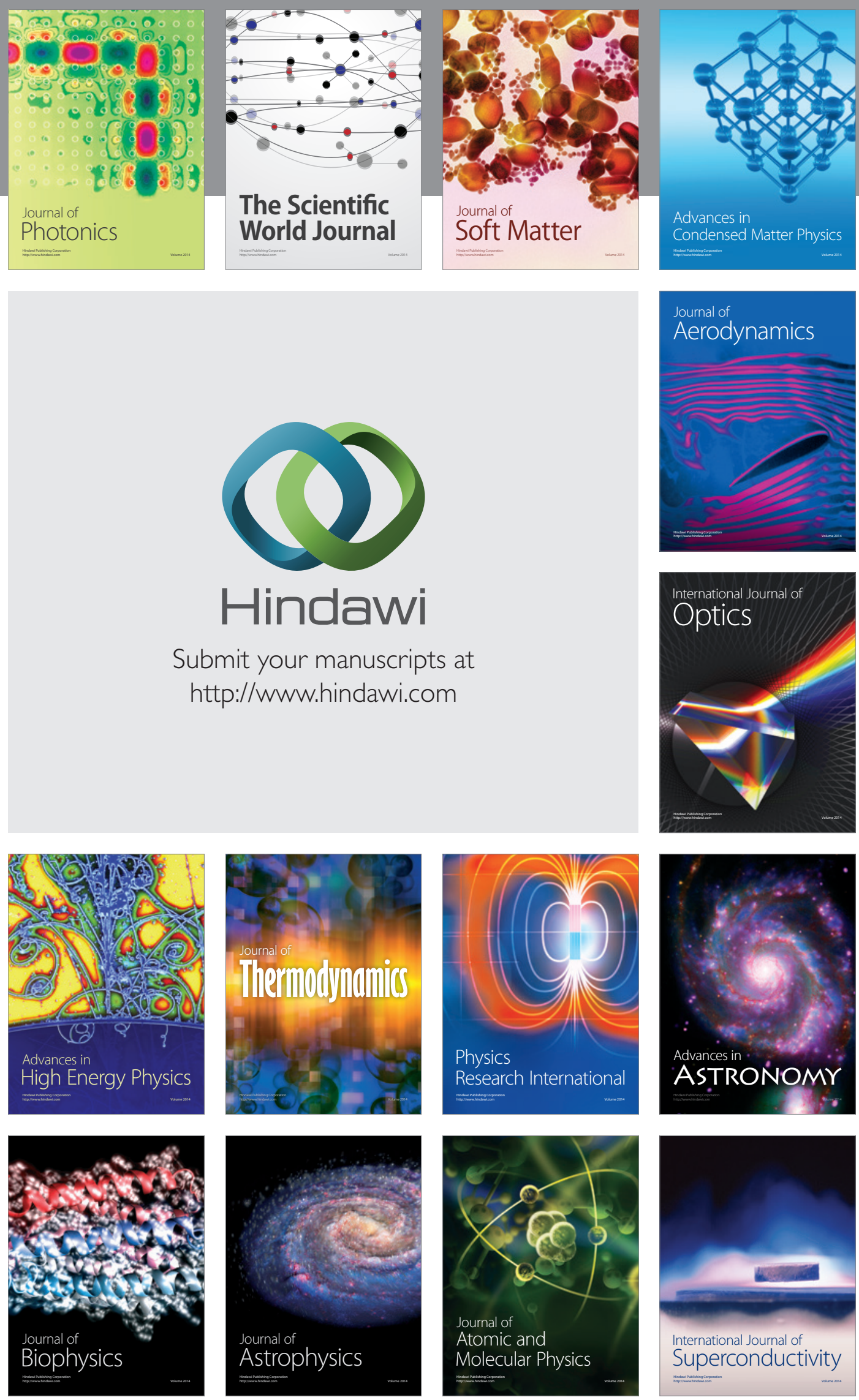
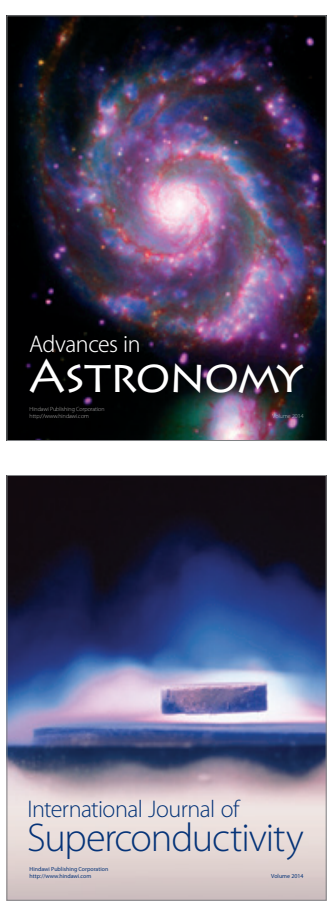